\title{
Tailor-made condensates
}

Keith Burnett

Bose-Einstein condensates are a strange form of ultracold matter, first created in the lab less than three years ago. A way has now been found to manipulate the interactions within condensates to an extraordinary degree, heralding previously impossible experiments and applications.

O ne of the favourite games of the theoretical physicist is to imagine that there is a little knob with which one can change the nature of the interactions between the atoms of a material. This is usually just a game, although it is a very instructive one: one sees very clearly in such an exercise how important the interaction strength is for the properties of real materials. For that reason it is something one would dearly like to be able to do in real life.

In new experiments at the Massachusetts Institute of Technology, reported on page 151 of this issue ${ }^{1}$, Wolfgang Ketterle's group has shown that, in Bose-Einstein condensates, the strength of the interactions between atoms can indeed be changed over a very wide range, and can even be switched from attractive to repulsive. So for ultracold trapped atoms, the theorists' dream has become reality. This is especially exciting because of the range of phenomena that can be studied as a consequence.

A Bose-Einstein condensed gas of atoms was first produced in 1995 by Eric Cornell, Carl Wieman and colleagues ${ }^{2}$ at JILA in the University of Colorado. They used evaporative cooling of atoms in a magnetic trap to achieve $\mathrm{it}^{2}$. In a Bose-Einstein condensate,

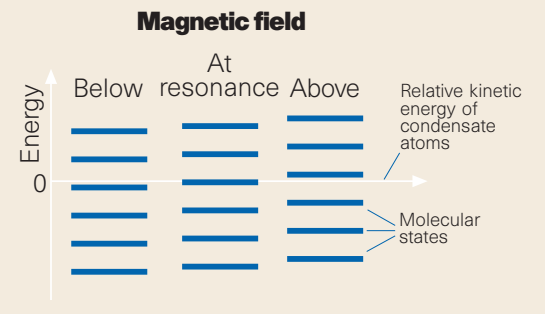

Figure 1 The energies of nearly-bound molecular states for different values of an applied magnetic field. These are states in which the atoms of a Bose-Einstein condensate hang together for some time instead of simply cruising past each other. Two atoms in the condensate are much more likely to form such a pair if their relative kinetic energy is close to the energy of the molecular state - in other words, if there is a resonance. Because a magnetic field alters the molecular-state energies, the strength of atom-atom interactions can be changed enormously by varying the field. all the atoms share the same quantum state, and it therefore has quite remarkable properties. In particular, it is subject to macroscopic quantum effects like those seen in superfluids, such as persistent viscosityfree flow. The production of such states was built on the development of laser cooling, for which the 1997 Nobel prize was awarded to Claude Cohen-Tannoudji, Steve Chu and Bill Phillips, and it is a remarkable technical achievement. But the properties of condensates are also of great interest to theorists, especially as atomic Bose condensates are so weakly interacting that first-principles microscopic calculations can be applied to their description.

The theoretical possibility of tuning the interaction between ultracold atoms using a
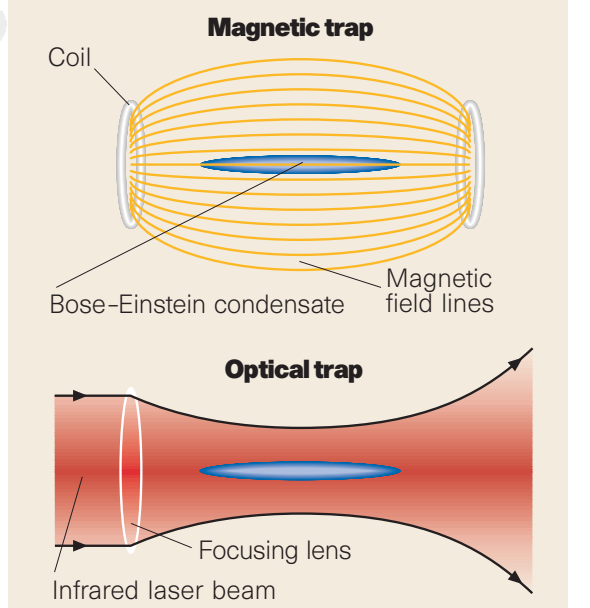

Infrared laser beam

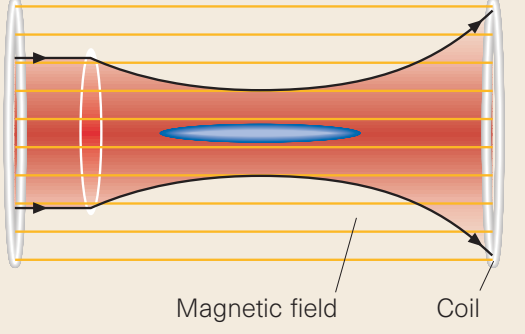

Figure 2 Trapping and tweaking a Bose-Einstein condensate. The condensate is first formed by evaporative and laser cooling in a magnetic trap (a). It is then contained by the optical field of a focused infrared laser (b), so that its interactions can be tuned by a uniform magnetic field (c). magnetic field was established some time ago $^{3}$. A modest magnetic field can distort the electron cloud around the atoms and hence change the force they exert on one another. This change is very small, but at the ultracold temperatures of the condensate it has a profound effect (Fig. 1).

To achieve this in the lab required the ability to trap the atoms so that they would not be too badly disturbed by a magnetic tuning field - the usual magnetic trapping methods clearly won't do. Instead, Ketterle et al. managed to trap their condensate in a light field. That isn't easy, as light fields easily destroy condensates unless the fields are closely controlled, and its accomplishment is a masterpiece of experimental technique.

Once they had made the condensate in a magnetic trap, the authors transferred it into an optical one, where the atoms are confined solely by the force a laser field exerts on an atom (Fig. 2). It is only possible to use such a trap because of the remarkably low temperatures, below $1 \mu \mathrm{K}$, achieved in the first step of creating the condensate. This makes the atoms slow enough for them to be held in a laser beam of modest power.

Ketterle and colleagues could then apply a near-uniform magnetic field (which does not unduly disturb the confinement of the atoms) and observe the changes this produced in the atomic interactions by the effect it has on the properties of the condensate. The changes they see are very marked, and the confirmation of theoretical predictions is impressive.

Being able to tune the interactions offers new ways to control the motion of condensed gases when they are allowed to escape to produce a laser-like source of atoms. By using a magnetic field with a varying field strength, one could focus the matter wave in a clean, loss-free manner. This should lead to more flexible sources of coherent matter waves for clocks, interferometry and lithography, for example.

This tuning knob will also let experimentalists play the game that theorists have been so very fond of in the past. One of the most exciting possibilities would be to form a large, stable condensate with repulsive interactions, and then to switch the interactions to being attractive. That would produce an unstable macroscopic quantum system that would rapidly collapse, ejecting hot atoms and molecules - providing a stringent test of the quantum field theory that is now being developed to treat condensates and their dynamics.

Keith Burnett is in the Clarendon Laboratory,

University of Oxford, Parks Road, Oxford OX1 3PU, UK.

e-mail:k.burnett1@physics.oxford.ac.uk

1. Inouye, S. et al. Nature 392, 151-154 (1998).

2. Anderson, M. H., Ensher, J. R., Matthews, M. R., Wieman, C. E. \& Cornell, E. A. Science 269, 198-201 (1995).

3. Davis, K. B. et al. Phys. Rev. Lett. 75, 3969-3973 (1995). 